\title{
Phase transition of two-dimensional Ising model on random point patterns
}

\author{
Xiujun Fu, ${ }^{1,2}$ Kwok Yip Szeto, ${ }^{1, *}$ and Wing Keung Cheung ${ }^{1}$ \\ ${ }^{1}$ Department of Physics, Hong Kong University of Science and Technology, Clear Water Bay, Hong Kong, China \\ ${ }^{2}$ Department of Physics, South China University of Technology, Guangzhou 510640, China \\ (Received 30 March 2004; revised manuscript received 28 July 2004; published 22 November 2004)
}

\begin{abstract}
The phase transition of two-dimensional Ising model on random point patterns is investigated using Monte Carlo simulation and the critical temperature is calculated using the Bethe approximation. We find a linear relation between the critical temperature and the structural characteristics of the random point pattern, as described by Aboav's parameter. Numerical results and analytical calculation both yield this linear relation with a similar slope, though the intercept is different due to the Bethe approximation.
\end{abstract}

DOI: 10.1103/PhysRevE.70.056123

PACS number(s): 05.50.+q, 82.70.Rr, 89.65.-s

\section{INTRODUCTION}

A common concern running through a wide range of disciplines is the examination of the spatial occurrence of a particular phenomenon. Illustrative examples that reflect the extensive range of scales covered by such phenomena include the location of sulfide inclusions in steel (metallurgy) [1], artifacts over a site (archaeology) [2], intensity of trees in a forest (ecology) [3], administrative systems in a province (geography) [4], and the distribution of galaxies in our universe (astronomy) [5]. In each of these instances, it is possible to represent individual incidences of the phenomenon as a set of $n$ points in space, defining what we called empirical point patterns [6]. These patterns represent one source of evidence that may be useful in learning more about the phenomenon and the processes responsible for creating them. In many different fields of science and engineering, many models have been constructed to derive hypotheses concerning the origin of the patterns. The pioneering work of Diggle [6] provides the complete spatial randomness (CSR) point pattern as a benchmark reference for many studies on the correlation generated by different physical or artificial processes. For example, the introduction of repulsion between points produces non-CSR patterns found in the works of Haggert et al. [7], Getis and Boots [8], Cliff and Ord [9], Ripley [10], and Upton and Fingleton [11]. Typically two measures are used for comparing the empirical point patterns with a given reference, namely the arrangement measure and the dispersion measure. The arrangement measure emphasizes characteristics that are invariant under translation, rotation, reflection, and changes of scale. The dispersion measures take into account the characteristic of the pattern that changes under these operations. Methods of analysis of the point patterns that have been widely used include the quadrate analysis, nearest-neighbor analysis, and second-order analysis [12]. However, very few works [13-20] address the topological characteristic of the point patterns. In this paper, we will employ some of the techniques developed in the study of soap froth evolution (a paradigm of twodimensional cellular patterns accessible to well-controlled

\footnotetext{
*Corresponding author. Email address: phszeto@ust.hk
}

experiments) to understand the topological characteristics of point patterns [13-17]. Here, we focus on point patterns generated with a given dynamics, which reflect the competition and cooperation of interacting agents in a two-dimensional cellular network, with each point being an individual agent.

Traditional studies of phase transition and critical phenomena for regular lattices and cellular automata for pattern evolution have provided us with many tools and insights in the analysis of the Voronoi network of a given point pattern. From the perspective of stochastic dynamical systems, the evolution of a point pattern with a well-defined equation of motion is an interesting subject of investigation in its own right. If there exists a stationary state emerging from the evolution, it will be more interesting as equilibrium statistical mechanics can be used for the analysis of the associated phase diagrams.

Having made these general remarks, we introduce two ideas for the studies of point patterns in two dimensions. The first one concerns the techniques of topological analysis. Topological analysis is a powerful tool in the understanding of two-dimensional cellular patterns. A common method of generating a two-dimensional cellular network for topological analysis from an empirical point pattern is to use the Voronoi construction, which dates back to Descartes' Principia Philosophiae in 1644. There are many applications of Voronoi diagrams. Besides the obvious use in the spatial analysis of two-dimensional patterns, there are many famous problems in spatial optimization that employ Voronoi construction. For example, the public mail boxes problem, which concerns the location of points so that the average distance to the nearest points is minimized; the minimization of average distance (or cost) to the nearest point on a service route provided that the total length of the route is given; the location of nodes (stations) and links (railways) to minimize the total flow cost (transportation cost) between any two given points on the plane; and the Steiner tree problem [21]. These problems in spatial optimization illustrate well the importance of topological characteristics of cellular patterns. We will use the shell model [13-16,20] and the concept of T1 neighbors [17] for a trivalent cellular network in the characterization of the structure. As these techniques are rather new, their application to the analysis of the point pattern coupled to a specific dynamics will be quite interesting. In this paper, we will only use the Aboav parameter [22] as a 
characterization tool of the random point patterns.

The second idea concerns the generalization of a traditional Hamiltonian of magnetism in the modeling of the dynamics to the point pattern, considered as a group of interacting agents constrained in a two-dimensional world. It is quite common in the studies of magnetism for a localized moment to be used for the quantum spin defined on a lattice, and for these local moments to interact with their neighbors with the Heisenberg model [23]. Here we do not have a lattice, but we have a network that provides us with a shell model with a clear meaning of its neighbors. Therefore, we can extend the idea of Heisenberg magnetism to point patterns. The formulation up to this point is a simple generalization of magnetism on a regular lattice to a network, not new in physics, though rather new to computer science [24-26]. However, the next step in the formulation is new to physics, but not to agent dynamics. We will assign a "quantum number" called color to each point and define a certain association of the point to the color. For example, color here can be interpreted as an indication of membership to a supermarket chain, though the individual member of the association is located in a different place on the plane, with their own states which carry other properties (other "quantum number") such as spin states. We see that in this formulation, the terms agent and point can be used interchangeably. An economic interpretation of this model is given by Szeto and Kong [27].

The dynamics governing the interaction of agents can be quite esoteric in real engineering applications and usually can be long range in space as well as in time [28-30]. Nevertheless, for the sake of simplicity, which is essential for physicists to achieve a deeper understanding of the point patterns, we limit this research to those interactions that are time-independent, and local in the sense that one can write a simple Hamiltonian to describe the dynamical system. This methodology of analysis couples the mathematics of the structure of a cellular network to traditional concepts in physics, with interaction taken out from computer science, which has more relevance to engineering and business application. This multidisciplinary approach therefore will carry with it important applications to other fields and the following discussion will outline the steps for the process of model building.

The simplest model makes use of only one "quantum number": the color of the agent. Supposing that we have only two colors, red and blue, then one of the simple Hamiltonians is

$$
H=\sum_{\langle i, j\rangle} J_{i j} S_{i} S_{j} .
$$

Here the interaction energy $J_{i j}$ is between the agent located at site $i$ with color $S_{i}$ and the agent located at site $j$ with color $S_{j}$. The summation is over all nearest neighbors. This is a generalized two-dimensional Ising model defined on a given nonregular lattice. One can introduce a Boltzmann factor based on this Hamiltonian to model the probability of the switching of color and perform Monte Carlo simulation to follow the evolution of the color pattern.
In this paper, we will first present the Monte Carlo simulation results of this Ising model in Sec. II. We then introduce the Bethe ansatz in solving the Ising model on twodimensional random point patterns in Sec. III. Finally, we will discuss the relation of the analytical work with the numerical simulation, with possible application to other fields.

\section{MONTE CARLO SIMULATION}

In a recent paper, we have generalized the Aboav-Weaire law to a cluster of cells in the shell model [14] for both experimental soap froth data and theoretical samples. Here we concentrate on the theoretical samples as they are much easier to control and generate. They are Voronoi patterns constructed from sets of $N$ points distributed randomly on a unit square. Different control levels on the minimal distance between any two points are used to generate different Voronoi patterns. We first define a cutoff distance $d_{\min }$ with $0 \leqslant d_{\text {min }}<1$, in the unit of $d_{\text {aver }}$, where $d_{\text {aver }}=1 / \sqrt{N}$ is the average distance between two points. Then, we sequentially place a point randomly on the unit square and calculate the minimum distance $d_{i}$ between this point $i$ and all other points on the square. If $d_{i} / d_{\text {aver }}>d_{\text {min }}$, we accept this point; otherwise we do not. This process continues until we get $N$ points on the square. We have generated 17 random point sets with $d_{\text {min }}$ from 0 to 0.8 in step of 0.05 . The Voronoi diagrams are then constructed based on these 17-point sets. The number of random points takes two values, $N=10000$ and $N=30000$, for all the 17-point sets. Except for the boundary ones, each point corresponds to a Voronoi cell and the number of Voronoi cells is about $96 \%$ of the original points. These large samples with $N=30000$ are used to get the Aboav parameters $a, b$, and self-consistent parameter $x$ [see Eqs. (4) and (5) below as well as Table I].

The Monte Carlo simulation is performed on Voronoi patterns with different $d_{\min }$ and free boundary conditions are employed. In order to reduce the boundary effect, we only analyze the central pieces of the large systems $(N=10000)$. The sizes of the cut pieces are $N=900,1600$, and 2500, respectively. The critical temperature in an infinite system is calculated by considering the fourth-order magnetization cumulant $U$ defined by [31]

$$
U(T, L)=1-\frac{\left\langle m^{4}\right\rangle}{3\left\langle m^{2}\right\rangle^{2}},
$$

where $L=\sqrt{N}$ is the linear size of the system and $m$ is the magnetization per spin. At critical temperature $U(T, L)=U^{*}$, which is independent of the system size $L$. Then the unique intersection point of $U$ for different $L$ provides an estimate of $T_{c}$. For each $L$, we do a simulation at the fixed temperature $T_{0}$ and make a measurement on a quantity $Q\left(\mathrm{~m}^{2}\right.$ or $\left.\mathrm{m}^{4}\right)$. By extrapolating to nearby temperatures $T$, the value of $Q(T)$ is obtained from the following expression $[32,33]$ :

$$
Q(T)=\frac{\sum_{i} Q_{i} \exp \left(\frac{1}{T_{0}}-\frac{1}{T}\right) E_{i}}{\sum_{i} \exp \left(\frac{1}{T_{0}}-\frac{1}{T}\right) E_{i}},
$$


TABLE I. Critical temperature and parameters of five Voronoi patterns. $a$ denotes the Aboav parameter and $b$ the intercept [see Eq. (4) below]. The self-consistent parameter $x$ used in the analytical calculation in Sec. III is also recorded [see Eq. (5)].

\begin{tabular}{cccccc}
\hline \hline & & & & $T_{c}$ & $T_{c}$ \\
$d_{\min }$ & $a$ & $b$ & $x$ & (Monte Carlo) & (Bethe ansatz) \\
\hline 0.0 & 0.6236 & 5.5979 & 6.2693 & $3.78 \pm 0.01$ & 5.205 \\
0.2 & 0.6953 & 5.8391 & 6.2404 & $3.75 \pm 0.01$ & 5.176 \\
0.4 & 0.8527 & 6.5199 & 6.1991 & $3.72 \pm 0.01$ & 5.134 \\
0.6 & 1.0592 & 7.3430 & 6.1373 & $3.68 \pm 0.01$ & 5.072 \\
0.8 & 1.2754 & 8.3078 & 6.0890 & $3.63 \pm 0.01$ & 5.023 \\
\hline \hline
\end{tabular}

where $Q_{i}$ is the $i$ th measurement of the quantity $Q$, and $E_{i}$ the corresponding energy. The simulation temperatures for the Voronoi patterns with $d_{\min }=0.0,0.2,0.4,0.6$, and 0.8 are, respectively, $T_{0}=3.80,3.75,3.70,3.65$, and 3.60 .

We use the Wolff algorithm [34] to perform Monte Carlo simulation. The flip times of all spins are accumulated and then divided by the total spin number, thus the Monte Carlo steps (MCS) per spin are calculated. At each temperature, the first 1000000 MCS per spin are discarded for equilibration. Then further 75000000 MCS per spin are performed for statistics. To determine the error bars, we split the configurations into 75 sets, each containing 1000000 configurations which are chosen from the simulation every $1000000 \mathrm{MCS}$ per spin. The critical temperature is calculated for each set and the errors are obtained by the standard method. In Table I, we show the geometric parameters for five Voronoi patterns and their corresponding critical temperatures obtained from the above Monte Carlo simulation. It can be seen that $T_{c}$ varies linearly with the Aboav parameter $a$. Also the results of $T_{c}$ deduced by the Bethe ansatz are listed, which will be discussed in the next section.

\section{BETHE'S APPROXIMATION IN VORONOI CELLULAR STRUCTURES}

We study the Ising model in Voronoi patterns by Bethe's approximation. Associated with each Voronoi cell is a spin variable $S_{i}$. There exist interactions between nearest neighbors only. In the absence of an external field, we introduce a cluster Hamiltonian of the form

$$
H(n)=-J \sum_{j=1}^{n} S_{0} S_{j}-B^{\prime}(n) \sum_{j=1}^{n} S_{j} .
$$

This cluster consists of a central cell with spin $S_{0}$ and its $n$ nearest neighbors with spins $S_{j}, j=1, \ldots, n$. The mean field $B^{\prime}(n)$ describes the average field acting on the cluster by its environment. The expectation values of the magnetization for the central spin and its neighbors are

$$
\begin{gathered}
f(n) \equiv\left\langle S_{0}\right\rangle, \\
g(n) \equiv\left\langle\frac{1}{n} \sum_{j=1}^{n} S_{j}\right\rangle .
\end{gathered}
$$

To be self-consistent, it is required that

$$
f(m)=g(n),
$$

where $m$ is the average number of sides of the neighboring cells which is related to the central cell with $n$ sides, by the Aboav-Weaire law

$$
m=6-a+\frac{b}{n} .
$$

Note that $m=m(n)$ depends on the number of edges, $n$, of the central cell. According to the definitions of Eqs. (1) and (2), $B^{\prime}(n), f(n)$, and $g(n)$ are also functions of $n$. As there is a probability distribution of cells with different sides in the Voronoi pattern, the physical quantities obtained will all depend on $n$. Therefore, it is reasonable to take $B^{\prime}(n)$ as a constant and let $m=n \equiv x$ to study the Ising model in a Voronoi structure. The value of $x$ is determined from Eq. (4),

$$
x=\frac{1}{2}\left[(6-a)+\sqrt{(6-a)^{2}+4 b}\right] .
$$

We see from this equation, which is a kind of "mean-field" approximation for the typical cell in the Voronoi pattern, that the number of edges of the typical cell is $x$, and is determined by the Aboav parameter $a$ and $b$. The partition function for the cluster is

$$
Q\left(x, B^{\prime}, T\right)=\sum_{\substack{S_{0}= \pm 1 \\\left\{S_{j}= \pm 1\right\}}} \exp \left[-H(x) / k_{B} T\right] .
$$

Denoting

$$
\alpha \equiv J / k_{B} T, \quad \alpha^{\prime} \equiv B^{\prime} / k_{B} T
$$

we can get the average value of magnetization for the central spin,

$$
f\left(x, \alpha, \alpha^{\prime}\right)=\frac{\left[2 \cosh \left(\alpha+\alpha^{\prime}\right)\right]^{x}-\left[2 \cosh \left(-\alpha+\alpha^{\prime}\right)\right]^{x}}{\left[2 \cosh \left(\alpha+\alpha^{\prime}\right)\right]^{x}+\left[2 \cosh \left(-\alpha+\alpha^{\prime}\right)\right]^{x}},
$$

and the average magnetization for the neighboring spins, 


$$
g\left(x, \alpha, \alpha^{\prime}\right)=\frac{\left[2 \cosh \left(\alpha+\alpha^{\prime}\right)\right]^{x} \tanh \left(\alpha+\alpha^{\prime}\right)+\left[2 \cosh \left(-\alpha+\alpha^{\prime}\right)\right]^{x} \tanh \left(-\alpha+\alpha^{\prime}\right)}{\left[2 \cosh \left(\alpha+\alpha^{\prime}\right)\right]^{x}+\left[2 \cosh \left(-\alpha+\alpha^{\prime}\right)\right]^{x}} .
$$

These are nonlinear equations of $\alpha$ and $\alpha^{\prime}$, and we need to see if there is a nonzero root for $\alpha^{\prime}$ so that the selfconsistency condition is satisfied, i.e.,

$$
f\left(x, \alpha, \alpha^{\prime}\right)=g\left(x, \alpha, \alpha^{\prime}\right) .
$$

We consider five different Voronoi patterns generated from $N$ random dots distributed in a unit square with a pair spacing constraint $d>d_{\text {min }}$. The geometric parameters of the five Voronoi networks are listed in Table I. To study the critical behavior of the Ising spins, we solve Eq. (10) numerically for each Voronoi system by setting $J=1$ and $k_{B}=1$. At the critical point, $\alpha^{\prime}$ has a nonzero solution and the average magnetization $\langle S\rangle$ tends to zero. The critical temperature versus the Voronoi parameter $d_{\min }$ and versus the Aboav parameter $a$ is plotted in Fig. 1. These calculations follow Huang [35] and agree with the simple relation $T c=2 J / k \ln [x /$ $(x-2)]$ for a regular lattice of coordination $x$. It can be seen from Fig. 1 that the critical temperature $T_{c}$ versus the
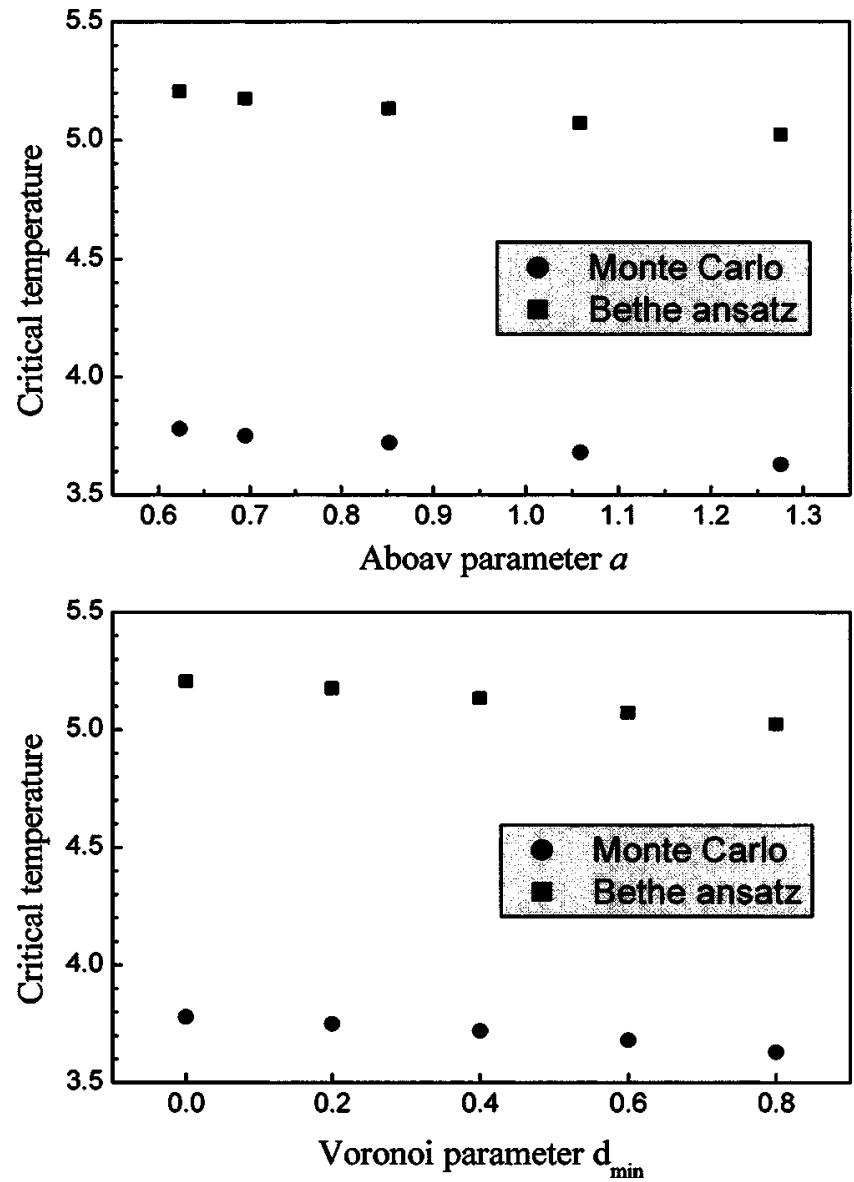

FIG. 1. The critical temperature vs Aboav parameter $a$ (upper) and vs Voronoi parameter $d_{\min }$ (lower) obtained from Monte Carlo simulation and from the Bethe ansatz.
Aboav parameter $a$ shows a linear relation. In the Bethe ansatz, the slope and intercept of the fitted straight line are -0.280 and 5.376 with error bars 0.0113 and 0.0105 , respectively. In the Monte Carlo simulation, the slope and intercept of the fitted straight line are -0.265 and 4.017 with error bars 0.0036 and 0.0034 , respectively.

Compared to Monte Carlo simulation results, we observed that although the values of $T_{c}$ have large difference because of the limitation of Bethe's approximation, the trend for different Aboav parameters is the same. The relative difference between $T_{c}$ for the Aboav parameter $d_{\min }=0.0$ and $d_{\min }=0.8$ from the Monte Carlo simulation is

$$
\frac{T_{c}\left(d_{\min }=0.8\right)}{T_{c}\left(d_{\min }=0.0\right)}=\frac{3.63}{3.78}=0.960 .
$$

Bethe's method gives

$$
\frac{T_{c}\left(d_{\min }=0.8\right)}{T_{c}\left(d_{\min }=0.0\right)}=\frac{5.023}{5.205}=0.965 .
$$

Based on this comparison, we can use the Bethe ansatz to predict the $T_{c}$ for a given Voronoi pattern, with appropriate adjustment for one of the two end points of this line.

\section{DISCUSSION}

We have computed the critical temperatures of the Ising model defined on various random point patterns using the Bethe ansatz. The analytical results exhibit a linear dependence of the critical temperature on the Aboav parameter, which can be used to predict the corresponding values from numerical simulation. The intercept of the linear relation is in error since the Bethe ansatz generally overestimates the magnitude of the critical temperature. Within the confine of the known limitation of the mean-field calculation of critical temperature, our analysis provides a simple and general method for estimating the critical temperature of all kinds of random point patterns. This is verified by Monte Carlo simulation here, but in general we expect that the linear relation between $T_{c}$ and the Aboav parameter holds, since $T_{c}$ is in general dependent on the effective coordination number, which is $x$ in our Bethe ansatz calculation. We see from Eq. (5) that the Aboav parameter enters into the calculation of $x$ quite naturally, since we expect a self-consistency in the account of the structure. Indeed, the application of the Aboav parameter allows us to couple the topological characteristics of the cellular pattern with the single cell approximation used in the mean-field calculation of the magnetization. We see that the trend shown in the Bethe ansatz for $T_{c}$ is a good approximation for the real $T_{c}$, as determined by numerical simulation. Thus, for a given random point pattern, we can compute first the Voronoi cellular structure based on this 
point pattern. Then by computing the Aboav parameter, we can read off the $T_{c}$ based on the theoretical calculation. Finally, the normalization on $T_{c}$ can be made using the offset of the Bethe ansatz for the hexagonal cellular structure, which corresponds to $d_{\min }=1$, as it is the $T_{c}$ for Ising spin defined on the lattice sites of a triangular lattice. In conclusion, our work shows that the $T_{c}$ for the Ising model defined on twodimensional random point patterns can be computed. This has application in econophysics as discussed in the paper by Szeto and Kong [27].

\section{ACKNOWLEDGMENT}

K.Y.S. acknowledges the support of RGC Grant No. HKUST 6157/01P and No. 6144/00P.
[1] W. A. Spitzig, J. F. Kelly, and O. Richmond, Metallography 18, 235 (1985); G. Burgers et al., in Advances in Phase Transition, edited by J. D. Embury and G. R. Purdy (Pergamon Press, Oxford, 1988), pp. 247-262.

[2] C. Renfrew, in The Explanation of Culture Change: Models in Prehistory, edited by C. Renfrew (University of Pittsburg Press, Pittsburgh, 1973), pp. 539-558.

[3] G. S. Brown, New Zealand Forestry Service Research Notes 38, 1 (1965).

[4] M. F. Goodchild and B. H. Massam, Geogr. Ann. 52B, 86 (1969).

[5] T. Matsuda and E. Shima, Prog. Theor. Phys. 71, 855 (1984).

[6] P. J. Diggle, Statistical Analysis of Spatial Point Patterns, (Academic Press, New York, 1983).

[7] Haggert, A. D. Cliff, and A. Frey, Locational Methods, (Edward Arnold, London, 1977).

[8] A. Getis and B. Boots, Model of Spatial Processes: An Approach to the Study of Point, Line and Area Patterns (Cambridge University Press, Cambridge, 1978).

[9] A. D. Cliff and J. K. Ord, Spatial Processes: Models and Applications, (Pion, London, 1981).

[10] B. D. Ripley, Spatial Statistics (John Wiley, New York, 1981).

[11] G. Upton and B. Fingleton, Spatial Data Analysis by Example. Volume 1: Point Pattern and Quantitative Data (John Wiley, Chichester, 1985).

[12] B. N. Boots and A. Getis, Point Pattern Analysis, Sage Scientific Geography Series, Vol. 8, (Sage Publications, Newbury Park, CA, 1988).

[13] K. Y. Szeto, Shell Model of Soap Froth, Proceedings of the Inauguration Conference of the Asia-Pacific Center of Theoretical Physics, Current Topics in Physics, Vol. 1, edited by Y. M. Cho, J. B. Hong, and C. N. Yang (World Scientific, Singapore, 1998), pp. 361-375.

[14] K. Y. Szeto, Xiujun Fu, and W. Y. Tam, Phys. Rev. Lett. 88, 138302 (2002).
[15] K. Y. Szeto, T. Aste, and W. Y. Tam, Phys. Rev. E 58, 2656 (1998).

[16] T. Aste, K. Y. Szeto, and W. Y. Tam, Phys. Rev. E 54, 5482 (1996).

[17] B. Dubertret, K. Y. Szeto, and W. Y. Tam, Europhys. Lett. 45, 143 (1999).

[18] G. LeCaer and R. Delannay, J. Phys. A 26, 3931 (1993); Phys. Rev. Lett. 73, 1553 (1994).

[19] N. Rivier, Philos. Mag. B 52, 795 (1985).

[20] M. A. Forte and P. Pina, Philos. Mag. B 67, 263 (1993).

[21] H. W. Kuhn, in Studies in Optimization, edited by G. B. Dantzig and B. C. Eaves, (Math. Assoc. Amer. Washington (1974), Vol. 10, p. 52.

[22] D. A. Aboav, Metallography 3, 383 (1970).

[23] D. C. Mattis, The Theory of Magnetism, (Springer-Verlag, Berlin, 1988).

[24] D. Espriu et al., Nucl. Phys. B 265, 92 (1986).

[25] H. Stüben, H.-C. Hege, and A. Nakamura, Phys. Lett. B 244, 473 (1990).

[26] W. Janke, M. Katoot, and R. Villanova, Phys. Rev. B 49, 9644 (1994).

[27] K. Y. Szeto and Chiwah Kong, Comput. Econ. 22, 163 (2003).

[28] Artificial Evolution: Third European Conference, AE '97, Nimes, France, 1997, selected papers, edited by J.-K. Hao et al. (Springer, Berlin, 1998).

[29] K. Binmore, J. Econ. Theory 80, 257 (1998).

[30] G. LeCaer and R. Delannay, J. Phys. A 26, 3931 (1993); Phys. Rev. Lett. 73, 1553 (1994).

[31] K. Binder, Z. Phys. B: Condens. Matter 43, 119 (1981).

[32] J. Glosli and M. Plischke, Can. J. Phys. 61, 1515 (1983).

[33] A. M. Ferrenberg and R. H. Swendsen, Comput. Phys. 3, 101 (1989).

[34] U. Wolff, Phys. Rev. Lett. 62, 361 (1989).

[35] K. Huang, Statistical Mechanics (Wiley Eastern, New Delhi,1988). 